\title{
Front Matter: Volume 8375
}

, "Front Matter: Volume 8375," Proc. SPIE 8375, Advanced Photon Counting Techniques VI, 837501 (15 June 2012); doi: 10.1117/12.979231

Event: SPIE Defense, Security, and Sensing, 2012, Baltimore, Maryland, United States 


\section{PROCEEDINGS OF SPIE}

\section{Advanced Photon Counting Techniques VI}

Mark A. Itzler

Joe C. Campbell

Editors

25-26 April 2012

Baltimore, Maryland, United States

Sponsored and Published by

SPIE

Volume $\mathbf{8 3 7 5}$

Proceedings of SPIE, 0277-786X, v. 8375

SPIE is an international society advancing an interdisciplinary approach to the science and application of light.

Advanced Photon Counting Techniques VI, edited by Mark A. Itzler, Joe C. Campbell, Proc. of SPIE Vol. 8375, 837501 - @ 2012 SPIE · CCC code: 0277-786X/12/\$18 - doi: 10.1117/12.979231 
The papers included in this volume were part of the technical conference cited on the cover and title page. Papers were selected and subject to review by the editors and conference program committee. Some conference presentations may not be available for publication. The papers published in these proceedings reflect the work and thoughts of the authors and are published herein as submitted. The publisher is not responsible for the validity of the information or for any outcomes resulting from reliance thereon.

Please use the following format to cite material from this book:

Author(s), "Title of Paper," in Advanced Photon Counting Techniques VI, edited by Mark A. Itzler, Joe C. Campbell, Proceedings of SPIE Vol. 8375 (SPIE, Bellingham, WA, 2012) Article CID Number.

ISSN 0277-786X

ISBN 9780819490537

Published by

SPIE

P.O. Box 10, Bellingham, Washington 98227-0010 USA

Telephone +1 3606763290 (Pacific Time) · Fax +1 3606471445

SPIE.org

Copyright () 2012, Society of Photo-Optical Instrumentation Engineers

Copying of material in this book for internal or personal use, or for the internal or personal use of specific clients, beyond the fair use provisions granted by the U.S. Copyright Law is authorized by SPIE subject to payment of copying fees. The Transactional Reporting Service base fee for this volume is $\$ 18.00$ per article (or portion thereof), which should be paid directly to the Copyright Clearance Center (CCC), 222 Rosewood Drive, Danvers, MA 01923. Payment may also be made electronically through CCC Online at copyright.com. Other copying for republication, resale, advertising or promotion, or any form of systematic or multiple reproduction of any material in this book is prohibited except with permission in writing from the publisher. The CCC fee code is 0277-786X/12/\$18.00.

Printed in the United States of America.

Publication of record for individual papers is online in the SPIE Digital Library.

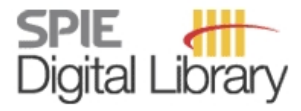

SPIEDigitalLibrary.org

Paper Numbering: Proceedings of SPIE follow an e-First publication model, with papers published first online and then in print and on CD-ROM. Papers are published as they are submitted and meet publication criteria. A unique, consistent, permanent citation identifier (CID) number is assigned to each article at the time of the first publication. Utilization of CIDs allows articles to be fully citable as soon as they are published online, and connects the same identifier to all online, print, and electronic versions of the publication. SPIE uses a six-digit CID article numbering system in which:

- The first four digits correspond to the SPIE volume number.

- The last two digits indicate publication order within the volume using a Base 36 numbering system employing both numerals and letters. These two-number sets start with 00, 01, 02, 03, 04 , $05,06,07,08,09,0 A, 0 B \ldots$. OZ, followed by 10-1Z, 20-2Z, etc.

The CID number appears on each page of the manuscript. The complete citation is used on the first page, and an abbreviated version on subsequent pages. Numbers in the index correspond to the last two digits of the six-digit CID number. 


\section{Contents}

vii Conference Committee

\section{PHOTON COUNTING FOR QUANTUM INFORMATION}

837503 Novel photon detection technologies for quantum communications (Invited Paper) [8375-02]

M. Sasaki, National Institute of Information and Communications Technology (Japan);

A. Tanaka, NEC Labs. America, Inc. (United States); M. Fujiwara, National Institute of Information and Communications Technology (Japan); K. Yoshino, S. Takahashi, Y. Nambu, NEC Corp. (Japan); A. Tomita, Hokkaido Univ. (Japan); A. Tajima, NEC Corp. (Japan); S. Miki, T. Yamashita, H. Terai, Z. Wang, National Institute of Information and Communications Technology (Japan); K. Tsujino, Japan Science and Technology Agency (Japan); D. Fukuda, National Institute of Advanced Industrial Science and Technology (Japan); M. Takeoka, National Institute of Information and Communications Technology (Japan)

837504 Quantum random number generators and their applications in cryptography (Invited Paper) [8375-03]

M. Stipčević, Univ. of California, Santa Barbara (United States) and Rudjer Boskovic Institute (Croatia)

837505 Reliable source of conditional states by multiple-photon subtraction using hybrid photodetectors (Invited Paper) [8375-04]

M. Bondani, Institute for Photonics and Nanotechnologies (Italy) and CNISM (Italy); A. Allevi, Univ. degli Studi dell'Insubria (Italy) and CNISM (Italy); M. G. Genoni, Imperial College London (United Kingdom); F. A. Beduini, ICFO - Institut de Ciències Fotòniques (Spain); S. Olivares, Univ. degli Studi di Milano (Italy) and CNISM (Italy) and Univ. degli Studi di Trieste (Italy); A. Andreoni, Univ. degli Studi dell'Insubria (Italy)

\section{APPLICATIONS OF PHOTON COUNTING}

837509 Functional analysis of fiber optic sensors using statistical photon counting: an automobile case study covered from quantum mechanics [8375-08]

J. A. Betancur, Univ. EAFIT (Colombia)

8375 OA Adaptive time-sequential binary sensing for high dynamic range imaging [8375-09] C. Hu, Y. M. LU, Harvard School of Engineering and Applied Sciences (United States)

PHOTON COUNTING ARRAYS FOR LIDAR IMAGING

8375 OC Photon counting ladar work at FOI, Sweden (Invited Paper) [8375-1 1]

O. Steinvall, L. Sjöqvist, M. Henriksson, Swedish Defence Research Agency (Sweden) 
8375 OD Geiger-mode APD camera system for single-photon 3D LADAR imaging [8375-12] M. Entwistle, M. A. Itzler, J. Chen, M. Owens, K. Patel, X. Jiang, K. Slomkowski, S. Rangwala, Princeton Lightwave, Inc. (United States)

8375 OE Low-power 20-meter 3D ranging SPAD camera based on continuous-wave indirect time-of-flight [8375-13]

S. Bellisai, L. Ferretti, F. Villa, A. Ruggeri, Politecnico di Milano (Italy); S. Tisa, Micro Photon Devices S.r.l. (Italy); A. Tosi, Politecnico di Milano (Italy); F. Zappa, Politecnico di Milano (Italy) and Micro Photon Devices S.r.l. (Italy)

\section{MULTIPHOTON COINCIDENCE COUNTING}

8375 OF An FPGA-based module for multiphoton coincidence counting (Invited Paper) [8375-14] D. Branning, Trinity College (United States); M. Beck, Whitman College (United States)

8375 Ol 4 channel, 20 ps resolution, monolithic time-to-amplitude converter for multichannel TCSPC systems [8375-17]

M. Crotti, I. Rech, I. Labanca, Politecnico di Milano (Italy); M. Ghioni, Politecnico di Milano (Italy) and Micro Photon Devices (Italy)

\section{SUPERCONDUCTING SINGLE PHOTON DETECTORS}

$83750 \mathrm{M}$ Experimental demonstration of a novel superconducting photon-number resolving detector at telecom wavelengths [8375-22]

S. Jahanmirinejad, G. Frucci, Technische Univ. Eindhoven (Netherlands); F. Mattioli, Istituto di Fotonica e Nanotecnologie (Italy); D. Sahin, Technische Univ. Eindhoven (Netherlands);

A. Gaggero, R. Leoni, Istituto di Fotonica e Nanotecnologie (Italy); A. Fiore, Technische Univ. Eindhoven (Netherlands)

\section{SINGLE PHOTON AVALANCHE DIODES}

8375 ON Silicon single-photon avalanche diodes for high-performance parallel photon timing (Invited Paper) [8375-23]

A. Gulinatti, I. Rech, C. Cammi, I. Labanca, Politecnico di Milano (Italy); P. Maccagnani, Istituto per la Microelettronica e Microsistemi (Italy); M. Ghioni, Politecnico di Milano (Italy) and Micro Photon Devices S.r.l. (Italy)

837500 Distortions from multi-photon triggering in a single CMOS SPAD (Invited Paper) [8375-24] M. W. Fishburn, E. Charbon, Delft Univ. of Technology (Netherlands)

8375 OP High-detection efficiency and picosecond timing compact detector modules with red-enhanced SPADs [8375-25]

A. Giudice, G. Simmerle, D. Veronese, R. Biasi, Micro Photon Devices (Italy); A. Gulinatti, I. Rech, Politecnico di Milano (Italy); M. Ghioni, Micro Photon Devices (Italy) and Politecnico di Milano (Italy); P. Maccagnani, IMM-CNR sezione di Bologna (Italy) 
$8375 \mathrm{OQ}$ A Ge-on-Si single-photon avalanche diode operating in Geiger mode at infrared wavelengths [8375-26]

M. Aminian, Ecole Polytechnique Fédérale de Lausanne (Switzerland); A. Sammak, L. Qi, L. K. Nanver, Technische Univ. Delft (Netherlands); E. Charbon, Ecole Polytechnique Fédérale de Lausanne (Switzerland) and Technische Univ. Delft (Netherlands)

8375 OR Development of small unit cell avalanche photodiodes for UV imaging applications [8375-27]

A. K. Sood, R. E. Welser, R. A. Richwine, Y. R. Puri, Magnolia Optical Technologies, Inc. (United States); R. D. Dupuis, J.-H. Ryou, Georgia Institute of Technology (United States); N. K. Dhar, DARPA/MTO (United States); P. Suvarna, F. Shahedipour-Sandvik, College of Nanoscale Science \& Engineering (United States)

\section{SOLID STATE PHOTOMULTIPLIERS AND NOVEL SPDS}

8375 OS Probabilistic analysis of solid state photomultiplier performance (Invited Paper) [8375-28] S. Vinogradov, P.N. Lebedev Physical Institute (Russian Federation)

8375 OT Photon-number statistics and correlations with silicon photomultipliers [8375-29]

A. Allevi, L. Nardo, Univ. degli Studi dell'Insubria (Italy) and CNISM (Italy); M. Ramilli, Univ. Hamburg (Germany) and Univ. degli Studi dell'Insubria (Italy); M. Bondani, Institute for Photonics and Nanotechnologies, CNR (Italy) and CNISM (Italy)

8375 OU InGaAs/InP negative-feedback avalanche diodes (NFADs) and solid state photomultipliers (SSPMs) [8375-30]

X. Jiang, M. A. Itzler, K. O'Donnell, M. Entwistle, K. Slomkowski, Princeton Lightwave, Inc. (United States)

HIGH-RATE PHOTON COUNTING WITH APDS

8375 OW Readout circuitry for continuous high-rate photon detection with arrays of InP Geiger-mode avalanche photodiodes (Invited Paper) [8375-33]

J. P. Frechette, P. J. Grossmann, D. E. Busacker, G. J. Jordy, E. K. Duerr, K. A. Mclntosh, D. C. Oakley, R. J. Bailey, A. C. Ruff, M. A. Brattain, J. E. Funk, J. G. MacDonald, S. Verghese, MIT Lincoln Lab. (United States)

8375 OY Linear photon-counting with HgCdTe APDs (Invited Paper) [8375-35]

G. Vojetta, F. Guellec, L. Mathieu, K. Foubert, CEA-LETI (France); P. Feautrier, IPAG (France); J. Rothman, CEA-LETI (France)

$83750 Z$ Avalanche discrimination and high-speed counting in periodically gated single-photon avalanche diodes (Invited Paper) [8375-36]

A. Restelli, J. C. Bienfang, Joint Quantum Institute (United States)

837510 Characterization of sinusoidal gating of InGaAs/InP single photon avalanche diodes [8375-37]

Z. Lu, X. Zheng, W. Sun, J. Campbell, Univ. of Virginia (United States); X. Jiang, M. A. Itzler, Princeton Lightwave, Inc. (United States) 
POSTER SESSION

837512 Picosecond-resolved FRET on non-amplified DNA for identifying individuals genetically susceptible to type-1 diabetes [8375-39]

L. Nardo, Univ. of Insubria (Italy) and CNISM (Italy); G. Tosi, Univ. of Insubria (Italy); M. Bondani, Institute for Photonics and Nanotechnologies (Italy) and CNISM (Italy);

R. Accolla, A. Andreoni, Univ. of Insubria (Italy)

Author Index 


\title{
Conference Committee
}

\author{
Symposium Chair
}

Kevin P. Meiners, Office of the Secretary of Defense (United States)

Symposium Cochair

Kenneth R. Israel, Lockheed Martin Corporation (United States)

Conference Chair

Mark A. Itzler, Princeton Lightwave, Inc. (United States)

Conference Cochair

Joe C. Campbell, University of Virginia (United States)

Program Committee

Gerald S. Buller, Heriot-Watt University (United Kingdom)

Sergio Cova, Politecnico di Milano (Italy)

William H. Farr, Jet Propulsion Laboratory (United States)

Robert H. Hadfield, Heriot-Watt University (United Kingdom)

Majeed M. Hayat, The University of New Mexico (United States)

Michael A. Krainak, NASA Goddard Space Flight Center (United States)

Robert A. Lamb, SELEX Galileo Ltd. (United Kingdom)

K. Alex McIntosh, MIT Lincoln Laboratory (United States)

Alan L. Migdall, National Institute of Standards and Technology (United States)

Michael Wahl, PicoQuant GmbH (Germany)

Hugo Zbinden, University of Geneva (Switzerland)

Session Chairs

1 Photon Counting for Quantum Information

Mark A. Itzler, Princeton Lightwave, Inc. (United States)

2 Single Photon Sources

Michael Wahl, PicoQuant GmbH (Germany)

3 Applications of Photon Counting

Michael Wahl, PicoQuant GmbH (Germany) 
$4 \quad$ Photon Counting Arrays for LIDAR Imaging

K. Alex McIntosh, MIT Lincoln Laboratory (United States)

$5 \quad$ Multiphoton Coincidence Counting

Alan L. Migdall, National Institute of Standards and Technology (United States)

$6 \quad$ Superconducting Single Photon Detectors

William H. Farr, Jet Propulsion Laboratory (United States)

$7 \quad$ Single Photon Avalanche Diodes

Joe C. Campbell, University of Virginia (United States)

8 Solid State Photomultipliers and Novel SPDs

Michael A. Krainak, NASA Goddard Space Flight Center (United States)

$9 \quad$ High-Rate Photon Counting with APDs

Hugo Zbinden, University of Geneva (Switzerland) 